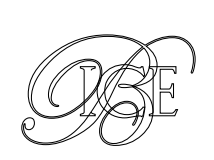

\title{
ANÁLISIS DE UNA NUEVA POLÍTICA PÚBLICA AL SERVICIO DEL DESARROLLO ECONÓMICO: Los programas de atracción de talento emprendedor extranjero (PATEX)
}

El presente artículo busca poner de relieve la importancia de la atracción del talento emprendedor internacional como una nueva expresión de política pública a través del estudio de los llamados Programas de Atracción de Talento Emprendedor Extranjero (PATEX). Para ello se realiza una revisión de la literatura económica referida al emprendimiento y la movilidad del talento, así como de los visados de emprendedores. Finalmente, se analizan los programas PATEX implementados en numerosos países en un contexto de creciente sofisticación y madurez de las políticas públicas de fomento del emprendimiento. Como conclusión, los programas de atracción de talento emprendedor abren un interesante espacio de trabajo en el ámbito de la política pública — y de la colaboración público-privada - para el fomento de la innovación, y evidencian la relevancia del papel de la Administración como compensador de fallos de mercado.

Palabras clave: desarrollo, innovación, entrepreneurship, movilidad.

Clasificación JEL: L26, F22, H41, H42, O38.

\section{Introducción}

El apoyo a los emprendedores se ha convertido con el cambio de siglo en una de las políticas públicas más extendidas para promover el desarrollo de los territorios. Son conocidos

*Adrián Blanco Estévez. Economista Experto en Economía Internacional y Políticas Públicas, MPM por Georgetown University. Gestor del programa Rising Startup Spain en España Exportación e Inversiones (ICEX).

** Iñaki Ortega Cachón. Doctor en Economía, director de Deusto Business School, profesor de la Universidad de Deusto y de la Universidad Internacional de La Rioja (UNIR).

Versión de abril de 2017. los pioneros casos de éxito de California en Estados Unidos o en el Estado de Israel, que han propiciado las mejores condiciones para que florezca el emprendimiento de alto impacto.

Por otro lado, es sabido que la movilidad de científicos es consustancial a las universidades desde su creación en la Edad Media. En la considerada universidad más antigua del mundo, la italiana de Bolonia, estudiaron ya en el siglo xv ilustres extranjeros como el polaco Nicolás Copérnico o Erasmo de Rotterdam. $\triangleright$ 
Esa «circulación de cerebros» se aceleró exponencialmente con las políticas implementadas en el siglo $x x$ a favor del movimiento de personas auspiciada por la expansión del libre comercio en todo el mundo.

Con la llegada de la llamada nueva economía, en la cual la ventaja competitiva migra hacia las actividades basadas en el conocimiento gracias al cambio tecnológico, ambos fenómenos descritos en los párrafos anteriores se están hibridando. Emprendimiento y libre circulación de talento son dos expresiones de esa nueva realidad económica que vivimos y que los Gobiernos de todo el mundo han empezado a aplicar en sus actuaciones.

Este artículo pretende explicar las nuevas políticas públicas de atracción de talento emprendedor que se han ido poniendo en marcha desde 2010 y multiplicado en los últimos años. Para ello, en primer lugar, se explicita el marco teórico que sustenta la intervención pública tanto a favor de los emprendedores como en beneficio de la atracción de talento. A continuación serán explicadas esas nuevas políticas con sus principales programas a lo largo y ancho del mundo, para terminar con una serie de lecciones aprendidas de su estudio.

\section{Desarrollo}

\subsection{Marco teórico de las políticas públicas de atracción de talento emprendedor}

Estudiaremos a continuación el estado del arte, los antecedentes de las políticas públicas, así como las últimas actuaciones públicas para los dos elementos claves que dan sentido a las nuevas acciones a favor de la atracción del talento emprendedor. Todo ello se resume en la Tabla 1.

\subsubsection{La importancia de los emprendedores}

\section{Estado del arte}

Cantillon (1755) es el primero que habla de los emprendedores. Para el economista irlandés, son empresarios que asumen riesgos en su intento de crear riqueza en un entorno de incertidumbre. Un siglo después, Marshall (1890), en Principles of Economics, destaca la actividad del empresario como proveedor de bienes para la sociedad, pero también de innovación y progreso. Afirma que la capacidad organizativa «era el cuarto factor productivo» detrás de la tierra, el capital físico y la mano de obra. $\triangleright$

TABLA 1

COMPARACIÓN ENTRE EMPRENDIMIENTOY LIBRE CIRCULACIÓN DE TALENTO

\begin{tabular}{|l|c|c|}
\hline & Emprendimiento & Circulación de talento \\
\hline Primeros autores & $\begin{array}{c}\text { Cantillon (1755) } \\
\text { Marshall (1890) }\end{array}$ & $\begin{array}{c}\text { Smith (1776) } \\
\text { Ricardo (1817) }\end{array}$ \\
\hline Autores modernos & $\begin{array}{c}\text { Baumol (1968) } \\
\text { Birch (1979) }\end{array}$ & Lida (2002) \\
\hline Antecedentes de las políticas & Leyes antitrust (s. xIx) & Becas Fullbright (1948) \\
\hline Primeras políticas & Small Business Act (1953) & Tratado de Maastricht (1992) \\
\hline Políticas más importantes & SBIR (1982) & Jovanovic (1982) \\
\hline Base conceptual del auge de las políticas & Startup Chile (2010) \\
\hline Políticas contemporáneas & Estatuto francés del emprendedor (2008) & Canadá VISA (2012) \\
\hline Políticas atracción talento emprendedor & & \\
\hline Fuente: elaboración propia. & &
\end{tabular}


El emprendedor, para Schumpeter (1911), es el factor más importante del desarrollo económico, porque en su búsqueda de beneficios introduce cambios e innovaciones que rompen el equilibrio y mueven el flujo circular de la economía hacia un estadio superior. Para el autor austriaco, el emprendedor no ha de ser necesariamente el empresario, sino que es aquella persona que en la empresa, no acomodándose a la situación establecida, introduce las innovaciones que finalmente, a través del proceso que denomina «destrucción creativa», provoca desarrollo económico.

Pero quien hace posible la explosión de la investigación científica en este terreno es Birch, con la publicación en 1979 de The job generation process. La presentación de este informe en el Congreso de Estados Unidos, en el que se ponía de manifiesto que la mitad de los nuevos puestos de trabajo en ese país fueron creados por nuevas empresas, tuvo un enorme eco entre políticos de todos los colores.

La base teórica que permite establecer la relación entre emprendimiento y crecimiento económico es provista por las nuevas teorías de la evolución de la industria de Jovanovic (1982). Frente a los que sugerían que los emprendedores retardaban la actividad económica (eran menos eficientes y estaban fuera de la actividad innovadora por falta de recursos), Jovanovic sugiere exactamente lo contrario: el emprendimiento estimula y genera crecimiento. La iniciativa emprendedora se convierte, por tanto, en el vehículo por el cual las ideas, en muchas ocasiones, son implementadas (Audretsch, 2002). La explicación que dan a este empoderamiento del entrepreneurship tiene su base en la globalización, que ha hecho migrar la ventaja competitiva hacia actividades basadas en el conocimiento. Los cambios tecnológicos han reducido el papel de las economías de escala, aumentado la competencia en los mercados y mejorado la capacitación de los agentes económicos, lo que ha beneficiado a las nuevas empresas.

\section{Las políticas públicas}

La demostración de la positiva relación de la actividad emprendedora con el empleo, el crecimiento, la productividad, la innovación y la cohesión social ha hecho que Gobiernos de toda ideología impulsaran esta figura con diferentes políticas. Las primeras actuaciones se sitúan en Estados Unidos con las leyes antitrust que no estuvieron motivadas únicamente por la preocupación del Gobierno federal de evitar comportamientos abusivos de la gran empresa sobre los consumidores, sino también sobre los pequeños negocios. En este sentido, la Ley Sherman de 1890, que prohibía la concentración empresarial bajo la forma de trust, es considerada la primera norma a favor de los emprendedores.

Aunque son muchas las actuaciones públicas que se desarrollan a partir de ese momento en todo el mundo, especialmente después de la Segunda Guerra Mundial, hay un hito en la política pública de estímulo empresarial al crearse, en Estados Unidos en 1982, un nuevo instrumento que facilitaría específicamente el surgimiento de empresas tecnológicas: Small Business Innovation Research Program (SBIR). Este programa obligaba a las principales agencias federales de I+D a destinar una parte de su presupuesto de investigación a la financiación de pequeñas empresas innovadoras. Tuvo un gran éxito en la financiación de proyectos innovadores, estando detrás del surgimiento de empresas como Intel, Apple o Compaq.

Otra política pública que merece ser mencionada es el Programa Yozma (1993) de Israel. Esta iniciativa tenía por objetivo desarrollar $\triangleright$ 
un sector del capital riesgo orientado hacia startups de alto componente tecnológico. A tal efecto, la Administración destinó cien millones de dólares para la constitución de diez fondos de capital riesgo en los que participarían también inversores privados, ascendiendo su aportación hasta el 60 por 100 de la cuantía total. El programa logró atraer a importantes vehículos financieros internacionales, interesados no solo por la posibilidad de invertir en proyectos de gran potencial, sino también por la opción de adquirir, al término de cinco años, la participación pública en los mismos a un precio fijado de antemano.

La crisis global de 2008 marca un impulso en esta intervención pública, vista por los Gobiernos como una eficaz estrategia de estímulo. En el campo de la eliminación de trabas fiscales y administrativas, destaca la Ley del Autoemprendedor de Francia de 2008, que crea una nueva forma mercantil con un régimen de IVA y de seguridad social específicos para las nuevas empresas. En cuanto a España, se han multiplicado las iniciativas que se dirigen a los nuevos empresarios, destacándose la Ley de apoyo a los emprendedores del año 2013. Latinoamérica no se ha quedado atrás y numerosos países han impulsado en los dos últimos años legislaciones a favor de los emprendedores, como es el caso de Argentina, México, República Dominicana o Guatemala.

Conviene destacar que el apoyo a los emprendedores está trascendiendo lo público para llegar a las grandes empresas. El emprendimiento corporativo, también conocido como intraemprendimiento o incluso innovación abierta, se ha convertido en los últimos cinco años en una de las estrategias más habituales de las grandes empresas. Buscan adoptar el modelo de éxito de innovación de las startups, y para ello han empezado a trabajar con ellas y/o convertir a los trabajadores en emprendedores (Ortega et al., 2017).

\subsubsection{La importancia de la atracción del talento}

\section{Estado del arte}

Las políticas públicas objeto de este artículo encuentran su sustento teórico en la literatura económica en favor del libre comercio, desde las primeras teorías (Smith, 1776; Ricardo, 1817) hasta las contemporáneas (Krugman, 1980; Porter, 1990). Muy cercana a esa defensa del comercio siempre ha estado la libre circulación de personas. Desde el surgimiento de las primeras universidades, los científicos e intelectuales se han desplazado por todo el mundo. Como señalan Meyer et al. (1998): el «nomadismo científico» es un fenómeno común en la comunidad de científicos e ingenieros. Richard Florida ha actualizado este nomadismo con su teoría de las clases creativas (2002). Para el profesor estadounidense, las áreas metropolitanas con alta concentración de trabajadores de tecnología punta se asocian con un nivel elevado de desarrollo económico. Florida postula que esas clases creativas urbanas fomentan un entorno personal y profesional abierto y dinámico que actúa como un polo de atracción de talento, empresas y capital. Sugiere que las políticas de fomento económico han de centrarse en atraer y retener talentos de alta calidad.

\section{Políticas públicas}

Los acuerdos de Bretton Woods de 1944 no solo fijaron un nuevo orden económico mundial para el periodo posterior a la Segunda Guerra Mundial con la creación del Banco Mundial y el Fondo Monetario Internacional, sino que $\triangleright$ 
también fueron el germen de una institución mundial que fomentase el libre comercio, organización que se creó en 1948 con el nombre de GATT. Este acuerdo se refundó en 1995 con la Organización Mundial del Comercio, que tenía como misión propiciar la libertad de comercio no solo en los bienes, sino también en los servicios y en la propiedad industrial y científica.

Unos años antes, en 1992, los países miembros de la zona de libre comercio europea, denominada CEE (Comunidad Económica Europea), firmaron el Tratado de Maastricht, en el cual el derecho de las personas a circular y residir libremente en la Unión Europea constituye la piedra angular, más allá de las consideraciones clásicas de carácter comercial.

Muy cerca en el tiempo y en el espacio de la reunión de Bretton Woods, un senador americano de apellido Fullbright convenció al Departamento de Estado para que proporcionara fondos para que los mejores expedientes europeos estudiaran, investigaran o ejercieran como docentes en Estados Unidos. El alcance inicial de estas ayudas, que nacen en 1948, se centró en Europa para evitar nuevas contiendas por la vía de los lazos afectivos, pero hoy el programa funciona en todo el mundo con el nombre de becas Fullbright. Este sistema ha sido implantado por multitud de países que buscan atraer a los mejores profesionales, generalmente en el ámbito científico y universitario (especialmente para luchar contra la conocida «fuga de cerebros» bautizada así en 1963 por la Royal Society ante la emigración de profesionales ingleses hacia América).

Por último, la madurez y la democratización del acceso a la tecnología han hecho posibles nuevas y eficaces políticas que hibridan los dos conceptos descritos anteriormente, emprendimiento y circulación de talento. Esas nuevas políticas se caracterizan por tener en cuenta la movilidad internacional del talento emprendedor, estar dirigidas a proyectos empresariales de fuerte componente innovador y tender puentes de colaboración con el sector privado. Esta reinvención de las actuaciones de apoyo a los emprendedores se explica en detalle en el apartado 3.

\section{Las nuevas políticas de atracción del talento emprendedor}

\subsection{El impacto de la atracción del talento emprendedor}

Existe abundante literatura económica que demuestra el impacto positivo de la atracción de emprendedores extranjeros sobre la economía y el tejido empresarial. En particular, dicho impacto se traduce en la introducción de modelos de negocio de carácter innovador, en la generación de nuevos nichos de empleo y, con carácter general, en un aumento de las capacidades empresariales (Borjas, 1986; Wadhwa et al., 2007; Hunt, 2011; Ribeiro-Soriano \& Mas Verdú, 2015). Además, existe evidencia de la mayor predisposición de los extranjeros en comparación con los nacionales a llevar a cabo nuevas actividades empresariales, tal y como demuestran estudios realizados por la OCDE (2012), que también concluyó que los emprendedores foráneos crean entre 1,4 y 2,1 empleos más que los locales. Los emprendedores extranjeros también han probado ser un posible contrapeso contra el declive demográfico, así como el económico por su capacidad de aceleración del proceso, y que, como hemos visto, Schumpeter bautizó como «destrucción creativa».

En Estados Unidos es, precisamente, donde el impacto positivo, que los emprendedores foráneos pueden ejercer sobre una economía, $D$ 
se ha manifestado con mayor intensidad. Se estima que más de la mitad (44 de 87) de las empresas estadounidenses con una valoración superior a los 1.000 millones de dólares fueron fundadas por inmigrantes. El valor conjunto de estas compañías es de 168.000 millones de dólares, volumen superior a la capitalización bursátil de los valores cotizados en México o Rusia. Por poner algunos ejemplos destacados, el ruso Sergei Brin es uno de los fundadores de Google; el francés Pierre Omidyar, de Ebay; o el canadiente Garrett Camp, de Uber. Otras empresas americanas que se han convertido en multinacionales líderes en sus sectores de actividad también tuvieron entre sus fundadores a no estadounidenses, como es el caso de Procter \& Gamble, Dupont, Colgate o Pfizer. El talento emprendedor extranjero fluye de forma natural hacia Estados Unidos debido a los evidentes factores de atractivo: abundancia de capital riesgo, talento tecnológico y técnico de primer nivel, un elevado número de startups con gran potencial de crecimiento, un clima empresarial altamente business friendly y centros de educación superior y de investigación de excelencia. No en vano, entre los veinte ecosistemas de emprendimiento más competitivos del mundo se sitúan siete localizaciones/ciudades estadounidenses: Silicon Valley, Nueva York, Boston, Los Ángeles, Seattle, Austin y Chicago.

\subsection{El antecedente: los visados para los emprendedores}

En los últimos años, distintos Gobiernos han incorporado la captación de talento en sus agendas y han puesto en marcha iniciativas para fomentar su atracción. Una de las más extendidas ha sido el establecimiento de condiciones especiales en la concesión de visados para emprendedores que solicitan la entrada al país para llevar a cabo una actividad empresarial, lo que ha desembocado en la creación de visados específicos para la actividad emprendedora. Ya a finales de los años sesenta del siglo pasado, países como Canadá y Australia establecieron sus primeros programas específicos de atracción de emprendedores, pero lo novedoso es la extensión alcanzada por esta figura en los últimos años. Aunque no existen estadísticas oficiales al respecto, a finales de 2015 al menos trece países contaban con esquemas de visados específicos para emprendedores extranjeros (Australia, Canadá, Chile, Dinamarca, España, Estados Unidos, Francia, Italia, Irlanda, Nueva Zelanda, Países Bajos, Reino Unido y Singapur).

Las diferencias fundamentales en el diseño de los visados para emprendedores entre unos países y otros residen en la obligatoriedad de un volumen determinado de inversión y de creación de puestos de trabajo, y en la duración del permiso de residencia y la concesión de la residencia permanente, entre otros. Además, algunos países establecen cuotas en el número de emprendedores extranjeros que buscan atraer cada año ${ }^{1}$. La tendencia generalizada en los últimos años a nivel internacional ha sido suavizar las condiciones exigidas a los emprendedores para la concesión del visado. No obstante, el auge del proteccionismo en Estados Unidos y en Europa podría revertir esta tendencia, como ya ha sucedido en el caso de la Administración Trump, con un endurecimiento de las condiciones de entrada.

\footnotetext{
1 Por ejemplo, el Gobierno canadiense concede únicamente 2.750 visados para emprendedores y el Gobierno de Nueva Zelanda solamente concede 400 global impact visa por año.
} 


\subsection{Nacimiento y extensión de los Programas de Atracción de Talento Emprendedor Extranjero (PATEX) ${ }^{2}$}

Aunque un emprendedor extranjero obtenga el visado para desplazarse a emprender a otro país, los obstáculos que sigue encontrando son relevantes, y superiores a los que encuentran los emprendedores nacionales. Por ejemplo, existen importantes restricciones de acceso a financiación derivadas del desconocimiento de las alternativas financieras en el país de destino o de la falta de historial crediticio o de colateral (Fairlie, 2013; Vincenza, 2014). Otra importante barrera de entrada es la escasa familiaridad con el entorno jurídico y regulatorio local, y con los aspectos formales e informales asociados a "hacer negocios». La ausencia de una red de contactos entre posibles proveedores, distribuidores o clientes, además del mayor riesgo percibido del emprendedor extranjero por parte de éstos, son otros obstáculos habituales.

En este contexto, y con el objetivo de contribuir a reducir estas barreras, así como de incrementar la oferta-país para los emprendedores extranjeros, algunos países han ido más allá de la puesta en marcha de visados para la atracción de talento emprendedor. En concreto, han diseñado programas más completos y ambiciosos basados en la provisión de un conjunto de servicios de soft landing ${ }^{3}$ de alto valor añadido a un número determinado de emprendedores o startups extranjeras. Estos programas de atracción del talento emprendedor extranjero son conocidos también por su acrónimo PATEX.

\footnotetext{
2 La lista de Programas de Atracción de Talento Emprendedor Extranjero (PATEX) mencionados en el artículo no es exhaustiva, sino que pretende reflejar ejemplos destacados implementados por distintos Gobiernos.

3 Anglicismo usado en el mundo de los negocios para referirse a la instalación de una empresa extranjera en un país sin apenas problemas.
}

En los PATEX, a través de un concurso público en concurrencia competitiva, a un número determinado de emprendedores o startups extranjeras ganadoras se les ofrece servicios de tramitación/concesión de visados, provisión de espacios de trabajo, asesoramiento para el proceso de establecimiento empresarial, asesoramiento enfocado al negocio o apertura a redes de contactos en el mercado local. Además, acostumbran a conceder un incentivo financiero, bien no reembolsable o a cambio de una participación en el capital de la nueva empresa constituida. Desde la perspectiva de la política pública, los programas PATEX, además de las ventajas asociadas a la introducción en el tejido productivo local de empresas innovadoras y lo que ello supone en aceleración del proceso de «destrucción creativa», cuentan con la ventaja de contribuir a la percepción de la imagen de país abierto al emprendimiento y la innovación en el exterior, además de generar deal flow 4 hacia el país promotor del programa. También permiten adaptar el programa a las necesidades o prioridades del país a través de la convocatoria. A su vez, el proceso de selección puede orientarse hacia un determinado sector o tipo de empresa que favorezca incluso a un determinado colectivo.

El diseño y puesta en marcha de este tipo de programas es un fenómeno reciente y que se ha implementado en un, hasta ahora, reducido grupo de países, impulsado principalmente por instituciones y Administraciones Públicas, pero en el que también se ha involucrado el sector privado. En la Unión Europea, países como Reino Unido, Francia y España ya han puesto en marcha programas PATEX. En Reino Unido, el programa Sirius ${ }^{5}$ es una $\triangle$

\footnotetext{
4 Anglicismo usado en el mundo del capital riesgo para referirse a la cartera de empresas susceptibles de ser invertidas por su atractivo.

5 http://www.siriusprogramme.com
} 
iniciativa creada en 2013 por el Department of International Trade (DIT), antiguo UK Trade and Investment (UKTI), a la que se han sumado diversas iniciativas británicas de apoyo al emprendimiento. El programa ofrece a un equipo de hasta tres miembros (uno de los cuales debe proceder de fuera del Reino Unido) una ayuda de 35.000 libras en financiación semilla y un conjunto de servicios valorados en 20.000 libras, que incluye espacio de trabajo y asesoramiento en una de las doce incubadoras colaboradoras del programa. A cambio, Sirius exige un 10 por 100 de la propiedad de los proyectos ganadores. El foco del programa son los recién graduados - hasta dos años máximo desde la finalización de los estudios superiores-y los proyectos en fase principalmente embrionaria. Hasta el momento el programa ha logrado atraer 2.300 emprendedores procedentes de 93 países a Reino Unido, que han creado 73 nuevas empresas.

En Francia se ha puesto en marcha en 2015 el French Tech Ticket $^{6}$, el programa más ambicioso de los existentes actualmente en Europa, tanto por su impacto esperado como por su dotación presupuestaria y el número de instituciones públicas involucradas en la iniciativa (Ministerio de Economía, Industria y Sector Digital, Ministerio de Interior, Ministerio de Asuntos Exteriores y Desarrollo Internacional, además de diversas agencias públicas como Business France o el Ayuntamiento de París). Los servicios ofrecidos en el paquete de landing a las startups ganadoras incluyen 45.000 euros por empresa, fast-track para la obtención del permiso de residencia, un programa de aceleración y doce meses de espacio de trabajo en una de las 41 incubadoras colaboradoras a lo largo de toda Francia. En la primera

6 http://www.frenchtechticket.com edición del French Tech Ticket, correspondiente al periodo 2015-2016, se seleccionaron a veintitrés startups entre 722 candidatas de más de cien países, y en la segunda edición, correspondiente al periodo 2017-2018, el programa se ampliará a setenta ganadoras.

Del mismo modo, en España se ha lanzado en 2016 un programa de atracción de talento emprendedor bajo el nombre de Spain Rising Startup, impulsado por la institución pública España Exportación e Inversiones (ICEX), y que cuenta con la colaboración de los ayuntamientos de Madrid y Barcelona y del programa Open Future de Telefónica a través de su aceleradora Wayra. La iniciativa ofrece a un máximo de quince startups ganadoras servicios de tramitación de visados, espacio de trabajo en las dos ciudades mencionadas, una contribución económica no reembolsable de 10.000 euros y servicios de asesoramiento adaptados a las necesidades de la empresa. El programa Spain Rising Startup está enfocado a startups que presenten cierta madurez y que tengan una respuesta del mercado positiva en su país de origen (facturación o clientes).

En América Latina, Puerto Rico ha puesto en marcha una iniciativa llamada Parallel18 ${ }^{7}$, que tiene como objetivo atraer startups al país para que hagan de este su plataforma para operar globalmente, especialmente hacia Estados Unidos. Parallel18 ofrece cinco meses del programa de aceleración a ochenta startups ganadoras, y una contribución financiera de 40.000 dólares. El aspecto diferencial de este programa es la introducción de la obligatoriedad a los emprendedores de establecer vínculos con universidades y centros educativos locales y realizar actividades de mentorización de alumnos. Otra experiencia latinoamericana interesante es $\triangleright$

\footnotetext{
7 www.parallel18.com
} 
el programa brasileño Seed ${ }^{8}$ (Startups and Entrepreneurship Ecosystem Development), una de las escasas iniciativas PATEX puestas en marcha por Gobiernos regionales ${ }^{9}$, concretamente por el Gobierno estatal de Minas Gerais, y que fue creado igualmente para atraer talento emprendedor extranjero a este estado brasileño. El programa Seed selecciona los cuarenta mejores proyectos empresariales de base innovadora, que reciben entre $68.000 \mathrm{y}$ 80.000 reales (entre 20.000 y 25.000 euros) en capital semilla. Además, se ofrece un periodo de estancia de seis meses en el que los proyectos serán apoyados para mejorar sus capacidades e impacto en el territorio.

En Asia, un ejemplo destacado es el de Corea del Sur, país en el cual el Gobierno ha puesto en marcha el programa K-Startup Grand Challenge ${ }^{10}$, cuyo objetivo es atraer a startups extranjeras para que utilicen el país como puerta de entrada y base operativa para abordar el mercado asiático. La iniciativa parte del Ministry of Science, ICT and Future Planning, de la National IT Industry Promotion Agency y otras instituciones públicas, y cuenta con el patrocinio de los grandes conglomerados industriales locales (chaebol) como Samsung, Hyundai o LG. El programa selecciona los veinte mejores proyectos a los que ofrece un incentivo financiero de 27.000 dólares, si bien los cuatro con mejor desempeño disponen de un incentivo adicional que puede alcanzar los 100.000 dólares. El K-Startup Grand Challenge ofrece servicios de visados, espacios de trabajo y aceleración, además de cubrir parte de los costes de alojamiento y vuelos derivados del desplazamiento a Corea del

\footnotetext{
8 http://www.minasdigital.mg.gov.br/inicio/projetos/projeto/36/ seed-en

9 En España, el Gobierno vasco lanzó, en 2016, un programa para atraer emprendedores de la industria $4.0 \mathrm{con}$ el nombre BIND4.0.

10 http://www.k-startupgc.org/
}

Sur de los emprendedores desde su país de origen. También, las startups tienen posibilidad de entrar en contacto y trabajar conjuntamente con los chaebol que patrocinan y participan en el programa.

Por último, un elevado número de países de reducido tamaño y población no cuentan con programas específicos para atraer talento extranjero, pero en su afán de convertirse en nodos de emprendimiento global, los programas públicos de apoyo al emprendimiento -no solamente de visados, sino también de financiación o de creación de empresas - tienen el foco y son muy utilizados por emprendedores extranjeros. Un ejemplo es Singapur ${ }^{11}$, país donde el 39 por 100 de sus 5,3 millones de habitantes ha nacido fuera de las 63 islas que conforman el país, y que ha hecho de la atracción de talento extranjero uno de los pilares de su competitividad económica. En Singapur, su competitivo entorno de negocios (ocupa la segunda posición en el informe Doing Business, solamente por detrás de Nueva Zelanda), un completo sistema de incentivos y coinversiones en startups por parte del Gobierno y su cercanía a Asia actúan como catalizadores para convertirlo en un polo de atracción de emprendedores extranjeros.

\subsection{Startup Chile: un ejemplo de PATEX pionero $^{12}$}

En América Latina, el programa Startup Chile, puesto en marcha por el Gobierno de Sebastián Piñera en 2010, es actualmente $\triangleright$

\footnotetext{
11 Las iniciativas públicas del Gobierno de Singapur de apoyo a las startups se engloban en el programa Startup SG, y pueden ser consultadas en https://www.spring.gov.sg/Nurturing-Startups/Pages/ nurturing-startups-overview.aspx

12 Los autores agradecen los valiosos comentarios y aportaciones de Sebastián Díaz, director de Marketing y Comunicación de Startup Chile.
} 
el más completo que existe no solamente en su región, sino en todo el mundo. El programa parte de la máxima autoridad del Gobierno, está gestionado por Corfo $^{13}$ y ha evolucionado notablemente desde su creación, introduciendo mejoras y adaptaciones para maximizar el impacto de las startups participantes en la economía y el tejido empresarial chileno. En su formato actual, Startup Chile ha especializado su oferta dependiendo del público objetivo y cuenta con tres programas diferenciados: The $S$ Factory, Seed y Scale.

The S Factory está basado en proyectos en fase inicial liderados por mujeres, y ofrece 15.000 dólares en financiación y un programa de aceleración de cuatro meses. El programa Seed tiene como objetivo la aceleración de proyectos con un producto/servicio desarrollado y que estén validados por el mercado, y ofrece 30.000 dólares en financiación y un proceso de aceleración de seis meses. Por último, el Scale está dirigido a startups más maduras cuyos modelos empresariales estén en fase de escalamiento, y ofrece hasta 90.000 dólares. Startup Chile va un paso por delante de otros programas PATEX y, además de los servicios de visados o asesoramiento, cuenta con otros servicios, como, por ejemplo, un club de inversores compuesto por noventa inversores privados y fondos de venture capital para facilitar su entrada en el capital de las startups que forman parte del programa.

Startup Chile también es el programa que ha sido más evaluado - tanto interna como externamente- y que ha desarrollado diferentes métricas para medir su desempeño: a finales

13 Corporación de Fomento de la Producción (Corfo) es la agencia del Gobierno de Chile dependiente del Ministerio de Economía, Fomento y Turismo, a cargo de apoyar el emprendimiento, la innovación y la competitividad. de 2016 las startups que habían formado parte del programa sumaban una valoración conjunta de 1.350 millones de dólares, habían levantado 420 millones de dólares de capital, multiplicando por diez lo invertido por Corfo a través del programa, y creado 1.562 puestos de trabajo en Chile.

\section{Conclusiones}

Las políticas públicas para los emprendedores acumulan más de cien años de prácticas; además, en lo que llevamos de siglo, se han convertido en uno de los recursos más usados por Gobiernos en todo el mundo para propiciar desarrollo económico. Sin la madurez de estas políticas nunca hubiera sido posible el surgimiento de una actuación tan sofisticada como la de los PATEX y su rápida extensión por muchos países, como hemos visto en el artículo.

Estos programas no solo han hibridado las actuaciones a favor de los emprendedores y la atracción de talento, sino que han incorporado la colaboración pública-privada. Los PATEX también marcan la tendencia del futuro de las políticas públicas de emprendimiento, como es el caso del emprendimiento corporativo puesto en marcha por muchas grandes empresas en todo el mundo con el apoyo del ecosistema emprendedor local (en el que lo público tiene y seguirá teniendo un papel clave).

A pesar de lo anterior, es preciso seguir actuando a favor de los emprendedores porque persisten muchos fallos de mercado - como la ausencia de financiación-y públicos - la excesiva burocracia, por ejemplo- que impiden que las nuevas empresas puedan aportar todo su potencial a favor del desarrollo económico y social de los territorios. 
El momento actual caracterizado por un auge del proteccionismo amenaza con revertir el camino andado en la apertura de fronteras al talento internacional. En este contexto, los PATEX cobran una mayor importancia como herramienta de política pública, que puede ejercer de contrapeso a esta tendencia para continuar potenciando y ampliando la movilidad internacional del talento emprendedor.

De cara al futuro persisten importantes retos. Los policymakers necesitan poner en valor este tipo de programas tanto dentro de la Administración como ante distintos stakeholders implicados en la actividad empresarial y por ende en el desarrollo económico. Además, los programas deben continuar avanzando y especializándose - por ejemplo, verticalizándose sectorialmente- para adaptarse a las necesidades de las startups extranjeras y optimizar el proceso de solf landing de las mismas. Por su parte, la academia deberá incorporar el análisis y la evaluación de este tipo de programas a la investigación económica, desarrollando métricas que permitan una mejor comprensión del impacto generado por los PATEX en términos de generación de riqueza, empleo e innovación.

\section{Bibliografía}

[1] AUDRETSCH, D.B. (2002). Entrepreneurship: A survey of the literature. Institute For Development Strategies. Indiana University \& Centre for economic policy. Research (Cepr). Prepared for the European Commission, enterprise directorate.

[2] ANDERSON, T. (2016). Immigrants and biIlion dollars startups. Arlington. Disponible en: http://nfap.com/wp-content/uploads/2016/03/ Immigrants-and-Billion-Dollar-Startups. NFAP-Policy-Brief.March-2016.pdf
[3] BAUMOL, W.J. (1968). «Entrepreneurhsip in economic theory». American Economic Review, vol. 58, n.․ 2, pp. 64-71.

[4] BIRCH, D.L. (1979). The job generation process. Cambridge, Ma: Mit Program On Neighborhood And Regional Change.

[5] BORJAS, G. (1986). «The self-employment experience of inmigrants". The Journal of Human Resources, vol. 21, n.ำ 4, pp. 485-506.

[6] CANTILLON, R. (1755). Essai sur la nature du comerse en general. Londres y París.

[7] EUROPEAN COMMISSION (2016). Proposal for a Directive of the European Parliament and the Council on the conditions of entry and residence of third-country nationals for the purposes of highly skilled employment. Estrasburgo. Disponible en: http:// eur-lex.europa.eu/legal-content/EN/TXT/ $P D F /$ ?uri=CELEX:52016SC0194\&from $=E N$

[8] FAIRLIE, R.W. (2013). International Handbook on the Economics of Migration. Massachusetts (Estados Unidos): Edward Elgar Publishing Limited.

[9] FLORIDA, R. (2002). The rise of the creative class. New York: Basic Books.

[10] GONZÁLEZ URIBE, J. (2015). El caso de Startup Chile. Programa de atracción de talento para fomentar el emprendimiento. Caracas. CAF. Banco de Desarrollo de América Latina. Disponible en: http://publicaciones.caf.com/ media/42997/chile_startup_programa_talento_caf.pdf

[11] HUNT, J. (2011). «Which Immigrants are Most Innovative and Entrepreneurial? Distinctions by Entry Visa». Journal of Labor Economics.

[12] HUNT, J., y GAUHTIER-LOISELLE (2010), «How much does inmigration boost innovation?». American Economic Journal: Macroeconomics, vol. 2.

[13] JOVANOVIC, B. (1982). "Selection and the evolution of industry». Econometrica, vol. 50, n. 3 , pp. 649-670.

[14] KRUGMAN, P. (1979). «Increasing returns, monopolistic competition and international trade». Journal of International Economics, vol. 9, n.․ 4, pp. 469-80.

[15] MARSHALL, F. (1890). Principles of economics. Londres: Macmillan.

[16] MEYER, J.; KAPLAN, D., y CHARUM, J. (1998). «El nomadismo científico y la nueva $D$ 
geopolítica del conocimiento». En Charum, J., y Meyer, J. B. (eds.), El nuevo nomadismo científico. La perspectiva latinoamericana. Bogotá: Escuela Superior de Administración Pública (ESAP).

[17] ORGANIZACIÓN PARA LA COOPERACIÓN Y EL DESARROLLO ECONÓMICO (OECD) (2012). Open for Business; Migrant Entrepreneurs in OECD Countries. París. Disponible en: http://www. seipa.edu.pl/s/p/ artykuly/91/917/OECD\%20Migrant\%20 Entrepreneurship\%202012.pdf

[18] ORTEGA CACHÓN, I.; DE PABLOS, I., y SOTO, I. (2017). El emprendimiento corporativo en España. CISE-GEM. Santander.

[19] PORTER, M. E. (1990). The Competitive Advantage of Nations. New York: Free Press. (Republished with a new introduction, 1998).

[20] RICARDO, D. (1821). On the principles of political economy, and taxation. John Murray.
[21] RIBEIRO SORIANO, D., y MAS-VERDU, F. (2015). Special Issue on: «Small business and entrepreneurship: their role in economic and social development». Entrepreneurship and Regional Development, vol. 27, pp. 255-257.

[22] SMITH, A. (1817). An Inquiry into the Nature and Causes of the Wealth of Nations, vol. 2. Рипол Классик.

[23] STARTUP GENOME (2017). Global Startup Ecosystem Report. San Francisco. Disponible en: https://startupgenome.com/

[24] VINCENZA DESIDERIO, M. (2014). Policies to Support Inmigrant Entrepreneurship. Washington DC: Trasatlantic Council of Migration. Disponible en: http://www.migrationpolicy.org/research/ policies-support-immigrant-entrepreneurship

[25] WADHWA, V.; RISSING, B.; SAXENIAN, A. L., y GEREFFI, G. (2007). Education, entrepreneurship and inmigration: America's MNew Inmigrant Entrepreneurs. Part II. Kauffman Foundation. 